\title{
Bernard-Soulier Syndrome: An Inherited Platelet Disorder
}

\author{
Sivajee Sengupta \\ B.R. Singh Hospital, E. Railway, Kolkata 700 014, West Bengal, India
}

KEY WORDS Inherited disease; haemostasis; platelet

ABSTRACT In the present paper, a rare, potentially fatal, inherited disease-Bernard-Soulier syndrome-which is an autosomal recessive disorder of platelet and a deficiency disorder of GP1b-IX-V complex has been reported. This complex in an important in normal megakaryocytopoiesis, platelet turnover and maintenance of normal morphology of platelets.

\section{INTRODUCTION}

Bernard-Soulier Syndrome is a rare, potentially fatal, inherited disease. The prevalence of this disease is less than 1 in 1,000,000 as estimated from the cases reported in the literature. ${ }^{4}$ The disease was recognized to be familial and transmitted in an autosomal incompletely recessive pattern- since heterozygous individuals show mild biological abnormalities, but are normal clinically. ${ }^{2}$ Autosomal dominant inheritance has been reported in one family. ${ }^{4,5}$

Bernard-Soulier syndrome was first recognized by two French haematologists, Jean Bernard and Jean Pierre Soulier in the year 1948 in a patient who has prolonged bleeding time, mild to moderate thrombocytopenia and very large platelets. Since then a large number of patients have been identified presenting similar clinical and haematological features.

In normal primary haemostasis the role of platelet is immense. The most important role of platelet is clot formation. Normally the vascular wall act as a barrier between blood and extravascular space. When a platelet meets a damaged blood vessel wall, it tries to repair this damage by clotting. When tissues or blood vessels are injured, collagen is exposed to the blood. Platelet can adhere to components of the subendothelium at the site of injury. Platelets in BernardSoulier syndrome are lacking a part of the platelet that is involved in the initial stages of clot formation resulting increased bleeding tendency. Platelet adhesion is initiated by an interaction between von Willebrand factor-a coagulation factor exposed on the subendothelium and the
Glycoprotein (GP) Ib-IX-V complex on the platelet surface.

The platelet plasma membrane is vital to the platelet's function. It contains a number of specific glycoprotein (GP) receptors, through which platelets interact with aggregating agents, inhibitors, coagulation factors etc. ${ }^{2}$ The Glycoprotein $\mathrm{Ib}-\mathrm{IX}-\mathrm{V}$ complex is composed of, four membrane spanning polypeptide subunits GP Ib $\alpha$, GP Ib $\beta$, GPIX and GP V. These glycoproteins are all members of leucine rich glycoprotein. GP Ib $\alpha$ is the largest and the only subunit shown to bind vWF and thrombin and initiate the clotting process. ${ }^{3}$ GP Ib $\alpha$ subunit also contains the sequence in its cytoplasmic domain that attaches the complex to the platelet cytoskeleton. Glycoprotein-V has been shown to play a role in forming a high affinity thrombin binding site within the complex. The exact roles of GP Ib $\beta$ and GP IX are not clear, but probably they are supporting the role of GP Ib $\alpha$. They are necessary for efficient processing and expression of GP Ib $\alpha$ on the plasma membrane ${ }^{3}$.

But the mechanism by which GP Ib $\beta$ and GP IX participate for surface expression of this functional complex is not well understood. Absence of GP Ib $\beta$ or GP IX increased the rate of GP Ib $\alpha$ degradation ${ }^{3}$. GP IX has been shown to function as a receptor for drug dependent antibodies receptor sites, which were found to be decreased in Bernard - Soulier syndrome ${ }^{2}$. GP Ib complex binds with a great affinity to vWF and does not require prior stimulation of the platelets to bind $v W F$ - which is important in initial stages of clot formation. GP-Ib-IX-V complex is the receptor that causes platelet to stick. When this receptor is absent platelet cannot stick.

\section{Pathogenesis and Pathology}

Mutations of GP Ib $\alpha$ GP Ib $\beta$ and GP IX genes can cause. Bernard Soulier syndrome by decreasing the amount of GP Ib $\alpha$ appearing on the cell surface. As yet no case of Bernard Soulier syndrome associated GP V mutation has been reported, because GP V-/- platelets are normal in 
size and expressed normal amount of GP Ib-IX that is functional in vWF binding and can initiate normal clotting process. ${ }^{7}$ The most common form of Bernard Soulier syndrome is a nonsense mutation where a STOP codon is wrongly inserted in to the middle of a gene, resulting a much shortened, truncated GP Ib $\alpha$ protein $^{5}$ and results in a total absence of GP Ib complex on platelet memberane.

Absence or decreased expression of GP IbIX-V complex on the surface of platelet membrane is the main defect in Bernard Soulier syndrome. Megakaryocytes also show the same defect. As a result of decreased expression there is deficient binding of vWF to platelet membrane at the site of vascular injury, resulting in defective platelet adhesion. The end result is increased bleeding tendency due to non formation of platelet plug at the site of injury.

Single mutations within genes encoding either subunits of GP Ib or GP IX have been associated with the Bernard Soulier syndrome and the net effect of these mutations is a functional loss of vWF binding to the complex. There is experimental proof by animal model that bleeding, thrombocytopenia and giant platelets are all a direct result of an absent GP Ib-IX-V receptor. Presence of giant platelets, which is an important characteristic of human Bernard Soulier syndrome also observed in peripherial blood smear taken from GP Ib $\alpha$ null animal models. ${ }^{9}$ Although several Bernard Soulier patients have been described whose platelets are large but contain relatively normal surface levels of the complex. Transmission electron microscopy revealed abnormal megakaryocytes in GP Ib $\alpha$ deficient mice. The demarcating membrane system of GP Ib $\alpha$ deficient megakaryocyes appeared "vacuolated" or "disordered" -it indicates that there is a close association between expression of GP Ib $\alpha$ receptor or entire complex and normal megakaryocytopoiesis or ability of circulating platelets to maintain their normal morphology. ${ }^{9}$

Another important characteristic of Bernard Soulier syndrome is abnormal prothrombin consumption test. Bernard Soulier platelets have been reported to be deficient in collagen induced coagulant activity and to be unable to bind factor $\mathrm{XI}^{8} / \mathrm{GP} \mathrm{Ib}-\mathrm{IX} \mathrm{V}$ complex has been reported to be the platelet binding site for high molecular weight kininogen and for factor XII. Abnormal prothrombin consumption test has been reported to be correctable by the addition of factor VIII. This finding is consistent with the GP Ib-IX V complex providing a low affinity receptor on platelet for $\mathrm{vWF}$ /factor VIII. Treatment of normal platelets with anti GP Ib $\alpha$ antibody that blocks vWF binding causes same type of abnormal prothrombin consumption test as seen in Bernard Soulier syndrome patients. ${ }^{4}$

\section{Clinical Manifestations}

Usually the patients presented with prolonged mucocutaneous bleeding which is the only consistent symptom, some time they are moderate, but bleeding can often be severe and even fatal in about $16 \%$ of cases ${ }^{1}$. Platelet count ranges from very low to marginally low or even normal. ${ }^{4}$ Characteristic feature is abnormal morphology of platelets. The size of the platelet is always increased, sometimes upto $30 \mu \mathrm{m}$ in diameter (Giant Platelets), and typical granulations are clustered in the center of the platelet giving a pseudolymphocytic appearance. ${ }^{2}$ Ultra structure of the affected platelets show non specific abnormalities, but a dilated canalicular system, prominent dense tubular system and vacuolization have been described. Bone marrow shows plenty of megakaryocytes. Under light microscopy megakaryocytes show no characteristic morphologic abnormality, but electron microscopic studies have demonstrated abnormalities in dense tubular system and vacuolization of the demarcation membrane system ${ }^{6}$.

Prolonged bleeding time may be marginal to more than 20 minutes ${ }^{4}$.

\section{Laboratory Findings}

Thrombocytopenia, morphological abnormal platelets in peripheral blood film. Functional platelet tests show a lack of aggregation in presence of ristocetin which is not corrected by the addition of normal plasma as seen in von Willebrand disease, delayed aggregation after stimulation by thrombin. ${ }^{2}$ Platelet aggregation test in response to other agonists are normal. Clot retraction test is also normal. ${ }^{4}$

Confirmation of the diagnosis can be done by demonstrating membrane glycoprotein expression and its abnormality. In clinical practice, accurate estimation of the amount of platelet GP Ib, is possible immunologically, by fluorocytrometry, by ELISA, surface lebeling of washed platelets followed by sodium dodecyl sulfate polyacryla- 
mide gel electrophoresis and autoradiography or immunoblotting of platelet lysates with specific antiplatelet glycoprotein antibodies ${ }^{4}$. Recently molecular biological studies have been performed to establish the abnormality resulting platelet defects and to explain the lack or very decreased expression of the complex in Bernard Soulier syndrome.

\section{Differential Diagnosis}

Various coagulopathies are often not easily distinguishable from Bernard Soulier syndrome demanding specialized tests or biochemical analysis for proper diagnosis. They are-MayHegglin Anomaly, von Willebrand disease, Idiopathic Thrombocytopenic Purpura, Gray Platelet Syndrome etc.

\section{Variant forms of Bernard Soulier syndrome}

Variant forms of Bernard Soulier syndrome are reported in which platelet functional studies show abnormal ristocetin aggregation test where concentration of glycoprotein Ib is normal but showing different types of qualitative abnormalities.

\section{CONCLUSION}

Studies of inherited thrombocytopathies have proved to be an important tool to understand platelet physiology at the molecular level. Different studies on Bernard Soulier platelets have expand our knowledge of the role of Glycoprotein Ib-IX-V complex in the initiation of coagulation process. Bernard Soulier syndrome is a rare autosomal recessive disorder of platelet, and is a deficiency disorder of GP Ib-IX-V complex. This complex is also important in normal megakaryo- cytopoiesis, platelet turnover and maintenance of normal morphology of platelets.

\section{ACKNOWLEDGEMENT}

It is a pleasure to acknowledge my debt to my daughter Sugopa Sengupta for the assistance in preparation of this brief review.

\section{REFERENCES}

1. Bellucci S, Tobeiem G, Coen JP 1983. Inherited Platelet disorders. Prog Hematol, 13: 223-268.

2. Bellucci S, Sessa, Caen JP 1999. Inherrited Platelet Disorders-Postgraduate Haematology. Hofforand Lewis Tuddenham-4th Ed 583-584.

3. Jing-fei Dong, Shan Gao, Jose A Lopez-Synthesis 1998. Assembly and intracellular transport of the platelet glycoprotein Ib-IX-V complex. Journal of Biological Chemistry, 273: 31449-31454.

4. Jose A, Lopez Robert K 1998. Andrews Vahid Affshar-Kharghan, Michael C Barandt-Bernard Soulier Syndrome. Blood, 91: 4397-4418.

5. Laural B, Ludlow, Barbara P, Schick Marcia L, Budarf Deborah A, Driscoll Elaine H, Zackai Alan Cohen, Barbara A 1996. Konkle-Identification of a mutation in a GATA binding site of the Platelet Giycoprotein $\mathrm{Ib} \beta$ Promoter Resulting in the Bernard Soulier Syndrom. The Journal of Biological Chemistry, 271: 22076-22080.

6. Levine Shiley Parker 1998. Qualltitative Disorders of platelet function-Wintrobe's. Clinical Hamato-logy-10th Ed., Vol. 2: 1662.

7. Vanitha Ramakrishnan, Peter S, Reeves, Francis DeGuzman, Usha Deshpande, Kathleen MinistriMadrid, Robert B.Du Bridge, David R. Phillips 1999. Increased thrombin responsiveness in platelets from mice lacking glycoprotein. $V-P N A S G, 96$ : 13336-13341

8. Walsin PN, Mills DC, Pareti FI, Stewart GJ, Macfarlane DE, Jonson MM, Egan JJ 1975. Hereditary giant platelet syndrome. Absence of collagen induced coagulant activity and deficiency of factor Xl binding to platelets-B.J. Haematol, 29: 639.

9. Ware Jerry, Russel, Susan, Ruggeri Zaverio M 2000. Generation and rescue of a murine model of Platelet dysfunction: The Bernard Soulier Syndrome. PNAS, 97: 2803-2808. 